\title{
Hemocyte-Mediated Phagocytosis in Crustaceans
}

\author{
Shan Liu ${ }^{1 \dagger}$, Shu-Cheng Zheng ${ }^{1 \dagger}$, Yan-Lian $\mathrm{Li}^{3}$, Jun Li ${ }^{4 *}$ and Hai-Peng Liu ${ }^{1,2 *}$ \\ 'State Key Laboratory of Marine Environmental Science, State-Province Joint Engineering Laboratory of Marine Bioproducts \\ and Technology, Xiamen University, Xiamen, China, ${ }^{2}$ Laboratory for Marine Biology and Biotechnology, Pilot National \\ Laboratory for Marine Science and Technology, Qingdao, China, ${ }^{3}$ Department of Life Science and Engineering, Jining \\ University, Qufu, China, ${ }^{4}$ School of Science and Medicine, Lake Superior State University, Sault Ste. Marie, MI, United States
}

\section{OPEN ACCESS}

Edited by:

Xinjiang Lu,

Ningbo University, China

Reviewed by:

Chenghua Li,

Ningbo University, China

Yueling Zhang,

Shantou University, China

*Correspondence:

Jun Li

jli@/ssu.edu

Hai-Peng Liu

haipengliu@xmu.edu.cn

${ }^{\dagger}$ These authors have contributed equally to this work

Specialty section: This article was submitted to Comparative Immunology, a section of the journal

Frontiers in Immunology

Received: 29 December 2019 Accepted: 03 February 2020

Published: 03 March 2020

Citation:

Liu S, Zheng S-C, Li Y-L, Li J and Liu H-P (2020) Hemocyte-Mediated

Phagocytosis in Crustaceans.

Front. Immunol. 11:268

doi: 10.3389/fimmu.2020.00268
Phagocytosis is an ancient, highly conserved process in all multicellular organisms, through which the host can protect itself against invading microorganisms and environmental particles, as well as remove self-apoptotic cells/cell debris to maintain tissue homeostasis. In crustacean, phagocytosis by hemocyte has also been well-recognized as a crucial defense mechanism for the host against infectious agents such as bacteria and viruses. In this review, we summarized the current knowledge of hemocyte-mediated phagocytosis, in particular focusing on the related receptors for recognition and internalization of pathogens as well as the downstream signal pathways and intracellular regulators involved in the process of hemocyte phagocytosis. We attempted to gain a deeper understanding of the phagocytic mechanism of different hemocytes and their contribution to the host defense immunity in crustaceans.

Keywords: phagocytosis, hemocyte, innate immunity, white spot syndrome virus, crustacean

\section{INTRODUCTION}

The fundamental theory of phagocytosis was first described by Élie Metchnikoff in 1882, which has been gradually established and well-understood over the past two centuries (1). Phagocytosis currently is described as an endocytic process that endogenous foreign particles or pathogens larger than $0.5 \mu \mathrm{m}$ were first recognized by phagocyte surface receptor and then uptaken and engulfed into a plasma-membrane device, known as phagosome, following initiation of a signaling cascade to generate phagolysosome by fusion of phagosome with lysosomes. Finally, particles or pathogens within the phagolysosome will be degraded and cleared by the hydrolytic enzymes $(2,3)$. Phagocytosis has been considered as an essential defense mechanism of immune response to pathogens among eukaryotes, which are also implicated in diverse physiological processes, including development, apoptotic, tissue repair, and host defense (4). Owing to its importance and contributions to the innate and adaptive immune function in relation to human and animal health, phagocytosis still remains of great interests to many scientists.

Nowadays, aquaculture industry has become one of the most important resources for providing the people of the premium animal proteins, and employments over the world, especially in China and many Southeast Asian countries (5). However, the rapid expansion and development of aquaculture industries has been significantly inhibited due to infectious diseases, the key challenge imposed to sustainable aquaculture in China. Particularly, Vibrio bacteria and white spot syndrome virus (WSSV) have been recognized as the main threats among these pathogens in crustacean $(6,7)$. However, no effective strategies are available so far to control the outbreak of infectious diseases in crustacean aquaculture due to lack of knowledge about host-pathogen interaction, in particular the poor understanding of the host defensing immune function in crustaceans. 
During the past few decades, hemocyte-mediated phagocytosis, as one of the most important innate cellular immune function, has also received great attention in crustacean, and a good progress in elucidating the involvement of hemocytemediated phagocytosis, as well as its protective roles and mechanisms, against bacterial and viral infections has been achieved. In this review, we summarized the recent progress about phagocytosis of pathogens by hemocyte in crustaceans, in particular focusing on the novel findings about related receptors for recognition and internalization of pathogens as well as the downstream signal pathways and intracellular regulators involved in the process of hemocyte phagocytosis. We attempted to gain a deeper understanding of the phagocytic mechanism of different hemocytes and their contribution to the host defense immunity in crustaceans, which will be beneficial for the establishment of potential effective strategies to control diseases caused by viruses and bacteria in crustacean industries.

\section{BIOLOGICAL CHARACTERISTICS OF HEMOCYTE}

Phagocytes occur in many species, with extreme variations in abundance, evolving from the most primitive unicellular organisms, such as amoeba Dictyostelium discoideum, to higher multicellular vertebrates. Phagocytes in higher vertebrates, like mammalian species, have been highly specified into multifarious phagocytic cells, including neutrophils, eosinophils, monocytes/macrophages, dendritic cells, and osteoclasts, and termed as professional phagocytes, whereas others, so-called non-professional phagocytes, mainly include epithelial cells (8). However, regarding invertebrates, such as insects and crustaceans, phagocytes are kindly equivalent to hemocytes, which not only are involved in encapsulation, coagulation, and melanization but also exhibit phagocytic activities (9). Based on limited knowledge on crustacean hemocytes, three distinct types of hemocytes, named as hyaline cells, semigranular cells, and granular cells, have been classified and identified in most of crustaceans mainly based on cell size, nuclear/cytoplasmic $(\mathrm{N} / \mathrm{C})$ ratio, and the number of intracellular granules $(10,11)$. Hyaline cells have been characterized by their smallest cell size, having none or very few cytoplasmic granules, and the highest N/C ratios, while granular cells are the biggest cells with lowest N/C ratios and filled with larger cytoplasmic granules within hemocytes. As for semigranular cells, they are featured as their middle cell size between granular and hyaline cells, with smaller N/C ratios, and contain more cytoplasmic granules, but the number of granules is less than that of granular cells. Further molecular markers have also been proposed in a previous study for distinguishing different hemocytes of signal crayfish Pacifastacus leniusculus, and their results showed the potential application of superoxide dismutase (SOD) and two-domain Kazal proteinase inhibitor (KPI) to differentiate the granular and semigranular cells, respectively; however, more detailed molecular evidences are still required to further confirm their specificity in hemocyte classification (12).
Given its essential role in innate immune system, hemocyte homeostasis is of great importance for the health of crustaceans. Actually, the crustacean hematopoiesis has been well-investigated and described in several species since it was first reported in the early 1800s, such as shore crab Carcinus maenas, the lobster Homarus americanus, and signal crayfish P. leniusculus [an extensive review is made available by Lin and Söderhäll (13)]. For example, a study on signal crayfish P. leniusculus indicated that their hematopoietic tissue (Hpt) contain at least five different cell types corresponding to various developmental stages of granular and semigranular cells (11). Type 1 cells may be the precursor stem cells for the different cell lineages, and type 2 cells may be the precursor of granular and semigranular cells, both of which are the main cell types in Hpt. Types 3 and 4 may be the precursors of granular cells, whereas type 5 cells may lead to differentiating to semigranular cells (13). For the phagocytic capacity of different subpopulation of hemocytes in insects, previous studies demonstrated that plasmatocytes are the main phagocytic hemocytes in Drosophila, while granular cell and plasmatocyte are the main phagocytic hemocytes in Lepidoptera (14). Similarly, different subpopulation of hemocytes in diverse species or even in the same species of crustaceans also exhibited variable phagocytic activities. For instance, the higher phagocytic capacity has been observed in the hyaline cells rather than granular and semigranular cells of C. maenas, Carcinus aestuarii (15), and Eriocheir sinensis (16, 17), while main phagocytic capabilities of granular and semigranular cells were observed in Macrobrachium rosenbergii, Penaeus monodon, and Cherax quadricarinatus (17-20). Higher phagocytic activities were also demonstrated in the semigranular cells of signal crayfish $P$. leniusculus. In contrast, the phagocytic activity of semigranular cells was weaker than that of granular cells in Scylla paramamosain (15). However, it is noteworthy to mention that different subpopulations of hemocytes seem to exhibit specific preferences in phagocytosis of different bacteria or viruses. For instance, Escherichia coli was mainly ingested and cleared by semigranular and granular cells, whereas WSSV was mostly ingested by semigranular cells in red claw crayfish $C$. quadricarinatus (20). Although phagocytosis has been found in different subpopulation of hemocytes in distinct species, thus far, it is still very difficult to clearly classify the high amounts of evolutionary diversity of crustaceans. Meanwhile, the differentiating and developmental mechanisms of different subpopulations of hemocytes are also unclear. Therefore, more researches especially focused on fundamental theories still need to better characterize the characteristics of subpopulations of hemocytes and their corresponding phagocytosis in crustaceans.

\section{RECEPTOR OR OPSONIN-MEDIATED PATHOGEN RECOGNITION}

In mammals, microorganisms are initially recognized by phagocyte receptors, including $\mathrm{Fc} \gamma$ receptor, complement receptor, fibronectin receptor ( $\alpha 5 \beta 1$ integrin) and launch phagocytosis (3). Furthermore, the process of phagocytosis can be facilitated once pathogens were coated with opsonins (known 
as opsonization) because both opsonins and pathogen-associated molecular patterns (PAMPs) on the surface of pathogens are in turn easily recognized by phagocyte receptors. Phagocytosis mediated by hemocytes has been a great contribution to the defense in crustaceans against various pathogens, including Vibrio parahaemolyticus, Vibrio harveyi, Staphylococcus aureus, Aeromonas hydrophila, and WSSV. The receptors on the surface of hemocytes, such as lectins, scavenger receptors, immunoglobulin-related protein, and fibrinogen-related protein, have been reported in relation to phagocytosis for fighting pathogens (21-24). In the following, we summarized and discussed the recent advances on the involvement of related receptors or opsonin of hemocytes and their mediating recognition of pathogens in crustaceans.

\section{Lectins}

Lectins are featured by carbohydrate recognition domain (CRD) that consists of $\sim 110-130$ amino acids and binds to carbohydrates. So far, many groups of lectins, including Ctype, F-type, I-type, L-type, M-type, P-type, R-type, chitinase-like lectins, ficolins, calnexin, galectins, and intelectins, have been identified in crustaceans (21). It has been reported that lectins can act as opsonin or receptors in crustaceans to participate in the phagocytosis of foreign pathogens, including several bacteria and WSSV (21).

The C-type lectins (CTLs) were well-characterized among these lectins and highly conserved in crustaceans, which also acted as receptors to bind and agglutinate bacteria or served as opsonins to promote the phagocytosis of bacteria and viruses. Until now, various CTLs have been found to be implicated in phagocytosis in shrimp, crayfish, and crab, including P. monodon, Litopenaeus vannamei, S. paramamosain, Fenneropenaeus chinensis, and Procambarus clarkii (Table 1). In P. monodon, PmLec was found not only to act as a pattern recognition receptor (PRR) to recognize E. coli through binding with lipopolysaccharide (LPS) but also to function as an opsonin to enhance hemocyte phagocytosis (25). Since then, several lectins have also been identified in L. vannamei, which exhibited distinct levels on phagocytic activity against bacteria (27). For instance, the phagocytic rates against $V$. parahaemolyticus were decreased to $8.3,4.5$, and $2.5 \%$ after silencing the genes of $L v$ CTL5, LvCTLU, and $L v$ LdlrCTL, respectively, in comparison with control groups. It is obvious that the phagocytic activity mediated by LvCTL5 was significantly higher than the other two CTLs. Similarly, both PcLec3 from P. clarkii and SpCTL-B from $S$. paramamosain could also promote the phagocytic activity of hemocytes against Vibrio anguillarum and V. parahemolyticus, respectively $(31,32)$. Meanwhile, the expression of phagocytosisrelated genes, like SpMyosin, SpRab5, and SpLAMP [lysosomalassociated membrane protein 1-like protein (LAMP)], could be enhanced by SpCTL-B.

Apart from C-type lectins, L-type lectins and galectins, another type of lectin, were also identified as important opsonins to promote phagocytosis against bacteria and viruses in crustaceans. Both MjLTL1 and galectin were found to be able to enhance agglutination activity for bacterial clearance and hemocytes phagocytosis against V. anguillarum in Marsupenaeus japonicus and L. vannamei, respectively $(34,35)$. However, more types of lectins from various crustacean species and the molecular mechanisms involved in phagocytosis are extremely lacking, which need to be further elucidated for better understanding of lectin-mediated phagocytosis in innate immunity in crustaceans.

\section{Scavenger Receptors}

Scavenger receptors (SRs) are transmembrane proteins that can bind to modified low-density lipoproteins (LDLs), a broad range of polyanionic ligands and cell wall components, which play an essential role in physiological or pathological processes, such as intracellular cargo transport and clearance of pathogen and apoptotic cell (43). SRs occur on the surface of professional phagocytes in both mammalian species and invertebrates, which can be divided into nine heterogeneous classes (A-I) on the basis of their structural diversities $(22,39)$. SRs generally mediate phagocytosis of non-opsonic pathogens through recognizing PAMPs, including LPS and lipoteichoic acid (LTA). More recently, one of the class B scavenger receptors (SR-Bs), designated as SpSR-B, was found to a be functional phagocytic receptor in the mud crab $S$. paramamosain. The SpSR-B recombinant protein could bind onto the surface of $S$. cerevisiae, V. parahaemolyticus, V. alginolyticus, A. hydrophila, E. coli, S. aureus, and $\beta$ streptococcus. Moreover, SpSR-B-mediated phagocytosis could promote the clearance of $V$. parahaemolyticus and $S$. aureus. Meanwhile, the expression of phagocytosis-related genes SpLamp, SpRab5, SpArp, and SpMyosin were significantly downregulated when the SpSR-B gene was knocked down in hemocytes (36). Other studies also proved that class B scavenger receptors (SRBs) could bind to $S$. aureus and enhance the phagocytotic rate to facilitate subsequent microbial clearance in M. japonicus and E. sinensis $(39,40)$. In E. sinensis, the phagocytic rate for $V$. parahaemolyticus was decreased from approximately 21 to $15 \%$ after silencing of the EsSR-B1 gene, while the phagocytic rate for $S$. aureus was decreased from $\sim 15$ to $-7 \%$. All the findings indicated that SRB-mediated phagocytosis of $S$. aureus, $V$. anguillarum, or $V$. parahaemolyticus was very variable with regard to various host species $(36,39)$.

In addition, the class $\mathrm{C}$ scavenger receptors (SR-Cs), similar to that of mammalian class A scavenger receptors (SR-As), specifically recognized LPS of Gram-negative bacteria (22). MjSRC, a class C scavenger receptor characterized from $M$. japonicus, was found to be able to mediate phagocytosis of WSSV by binding the viral envelope protein VP19 and then restrict viral replication (37). Meanwhile, MjSRC could also act as a phagocytotic receptor for enhancing phagocytosis and restricting bacterial proliferation through recognizing V. anguillarum and $S$. aureus using its extracellular domains to bind bacterial polysaccharides, such as LPS and LTA (38). Owing to abundant heterogeneous classes of the SRs, it is worthy to further prove whether any other classes of SRs, besides SR-Bs and SR-Cs, are participating in hemocyte phagocytosis in crustaceans against bacterial or viral infection.

\section{Down Syndrome Cell Adhesion Molecule}

Owing to the lack of adaptive immune system for invertebrates, it is commonly accepted that the crustaceans are solely dependent 
TABLE 1 | Phagocytic receptors, ligands, and hemocyte-mediated phagocytosis of pathogens in crustaceans in previous studies.

\begin{tabular}{|c|c|c|c|c|c|c|}
\hline Receptors & Isoforms & Domains & Ligands & $\begin{array}{l}\text { Hemocyte-mediated } \\
\text { phagocytosis of } \\
\text { pathogens }\end{array}$ & Species & References \\
\hline \multirow[t]{9}{*}{ C-type lectin } & PmLec & CRD, QPD & LPS & E. coli & P. monodon & (25) \\
\hline & LvLec & CRD & - & V. alginolyticus & L. vannamei & (26) \\
\hline & LvCTL5 & CRD & - & V. parahaemolyticus & L. vannamei & $(27)$ \\
\hline & LVCTLU & CRD & - & V. parahaemolyticus & L. vannamei & (28) \\
\hline & LvLdlrCTL & CRD, LDLR & - & V. parahaemolyticus & L. vannamei & (29) \\
\hline & FCLec4 & QPD & - & V. anguillarum & F. chinensis & (30) \\
\hline & SpCTL-B & CRD & - & V. parahaemolyticus & S. paramamosain & (31) \\
\hline & PCLec3 & CRD, lg & LPS, PGN, LTA & V. anguillarum & P. clarkii & (32) \\
\hline & PCLT & CRD & $\begin{array}{l}\text { Envelope protein VP28, } \\
\text { VP19 }\end{array}$ & V. alginolyticus & P. clarkii & (33) \\
\hline L-type lectin & MjLTL1 & CRD & LPS, PGN, LTA & V. anguillarum & M. japonicus & (34) \\
\hline Galectins & LvGal & CRD & - & V. anguillarum & L. vannamei & (35) \\
\hline \multirow[t]{4}{*}{$\begin{array}{l}\text { Scavenger } \\
\text { receptors }\end{array}$} & SpSRB & CD36 & - & $\begin{array}{l}\text { V. parahaemolyticus } \\
\text { and S. aureus }\end{array}$ & S. paramamosain & (36) \\
\hline & MjSRC & MAM, CCP & $\begin{array}{l}\text { LPS, LTA, Envelope } \\
\text { protein VP19 }\end{array}$ & $\begin{array}{l}\text { WSSV, } V \text {. anguillarum } \\
\text { and S. aureus }\end{array}$ & M. japonicus & $(37,38)$ \\
\hline & MjSRB1 & CD36 & LPS, LTA & $\begin{array}{l}\text { V. anguillarum and } S . \\
\text { aureus }\end{array}$ & M. japonicus & (39) \\
\hline & EsSRB1 & CD36 & - & $\begin{array}{l}\text { V. parahaemolyticus } \\
\text { and S. aureus }\end{array}$ & E. sinensis & $(40)$ \\
\hline $\begin{array}{l}\text { Immunoglobulin- } \\
\text { related } \\
\text { proteins }\end{array}$ & EsDscam & $\lg , \mathrm{FN}$ & - & $\begin{array}{l}\text { V. parahaemolyticus } \\
\text { and S. aureus }\end{array}$ & E. sinensis & $(41)$ \\
\hline $\begin{array}{l}\text { Fibrinogen- } \\
\text { related } \\
\text { proteins }\end{array}$ & MjFREP2 & FReD & $\begin{array}{l}\text { LPS, PGN, envelope } \\
\text { protein VP28 }\end{array}$ & $\begin{array}{l}V . \text { anguillarum and } S . \\
\text { aureus }\end{array}$ & M. japonicus & $(42)$ \\
\hline
\end{tabular}

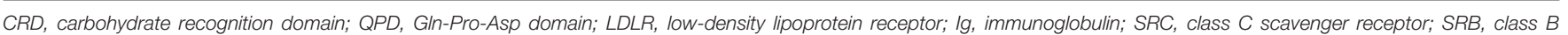

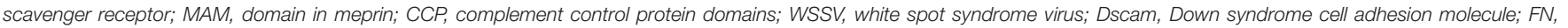
fibronectin; FReD, fibrinogen-related domain; LPS, lipopolysaccharide; PGN, peptidoglycan; LTA, lipoteichoic acid; CD36, cluster of differentiation 36; -, not available.

on innate immunity for the organisms against microbial infections. However, a highly variable immunoglobulin-related protein, named as Down syndrome cell adhesion molecule (Dscam), has been identified in both insects and crustaceans (24). As an immunoglobulin super family (IgSF) member, Dscam is composed of a cluster of immunoglobulin (Ig) and fibronectin (FN) domains (37). The first arthropod Dscam, which mostly presents in the neural system, fat body cells, hemocytes, and hemolymph serum in D. melanogaster (DmDscam), has been demonstrated as a phagocytic receptor (44). The Drosophila Dscam can generate more than 38,000 distinct extracellular domains by mutually exclusive alternative splicing of exons and potentially act as phagocytic receptors and/or opsonin for the recognition of various pathogens. In addition, the phagocytic activity of hemocytes was significantly decreased if the DmDscam gene expression was silenced in hemocytes (44). Similar to DmDscam, Dscams identified in Anopheles gambiae and Daphnia pulex can generate 31,920 and 13,312 alternatives splicing forms, respectively. After silencing of their Dscam gene in $A$. gambiae (AgDscam), the phagocytic capacity of the Sua5B cells was suppressed against E. coli and S. aureus. In crustacean, significantly high diversities of Dscam, generated by a variety of alternative splicing form repertoires with combination of three highly hypervariable Ig domain 2, 3, and 7, have been reported in L. vannamei, P. mondon, C. quadricarinatus, and E. sinensis $(41,45-47)$. For instance, the variable regions of $L v$ Dscam located in the Ig2, Ig3, and Ig7 domain could potentially encode at least 8,970 unique isoforms, which are speculated to play active roles in response to a spectrum of pathogens (47).

More recently, the EsDscam identified from Chinese mitten crab (E. sinensis) could potentially produce over 30,600 isoforms, and certain EsDscam isoforms were found to specifically bind with S. aureus, Bacillus subtilis, A. hydrophila, and $V$. parahaemolyticus, respectively, and then could facilitate efficient clearance of the bacteria through phagocytosis. Further investigations revealed that EsDscam contains two forms: soluble Dscam (Es-sDscam) and membrane-bound Dscam (EsmDscam). Like an opsonin, coating of Es-sDscam onto specific bacteria, such as $S$. aureus and $V$. parahemolyticus, could enhance the phagocytic ingesting of these bacteria, while such promoting phagocytic function was abolished for the truncated recombinant $E s$-sDscam. With respect to Es-mDscam, it served as a phagocytic receptor for Es-sDscam through interacting with the same isoform of Es-sDscam. In addition, studies have been 
demonstrated that higher expression level of EsDscam is in parallel to stronger and longer lasting phagocytic capacity of hemocytes in the immune priming E. sinensis with exposure to $A$. hydrophila (48). However, the underlying molecular mechanism of Dscam-mediated phagocytosis as well as its functioning regulation in response to bacterial and viral infections remain insufficient at present. Importantly, whether arthropod Dscam can serve as an antibody analog during the innate immune response against pathogenic infection, especially when we considered about the biological and functional specificity of a pathogen, if there is, remained to be further studied.

\section{Fibrinogen-Related Proteins}

Fibrinogen-related proteins (FREPs, also known as FBNs), containing a highly conserved fibrinogen-related domain (FReD), are one family of the pattern recognition receptors (PRRs) that consist of 53 putative members in A. gambiae, while only 20 known FREP members in D. melanogaster (49). The A. gambiae FREPs family has been proven to play a crucial role in mosquito against bacteria and malaria parasites, in which FREPs could bind with E. coli, Pseudomonas veronii, and Beauveria bassiana. FBN9 can form dimers for binding to the bacterial surfaces with different affinities (23). FREPs have also been reported to play an important role in the innate immunity of crustaceans. For instance, fibrinogen-related proteins were identified from M. japonicus (MjFREP1 and MjFREP2) $(19,42)$, which exhibited different expression levels in different tissues. Both MjFREP1 and MjFREP2 could bind to $V$. anguillarum and $S$. aureus through interaction with LPS and peptidoglycan (PGN). Furthermore, MjFREP2 could recognize the invading bacteria and facilitate bacterial clearance by promoting the phagocytosis of hemocytes. However, the molecular mechanism underlying hemocyte phagocytosis mediated by FREPs still needs further investigations, in consideration to their key roles in innate immunity.

\section{SIGNALING CASCADES INVOLVED IN PHAGOSOME FORMATION AND MATURATION}

After coating with opsonin and recognition of the pathogens by related phagocytic receptors, a series of signaling events for initiating phagocytosis will be thereby triggered, and will result in remodeling of the actin cytoskeleton to produce membrane pseudopods for internalizing the pathogen to form phagosome (50). Then, phagosomes gradually fuse with early and late endosomes and eventually fuse with lysosome to generate phagolysosome. Importantly, small GTPases of the Rab family represent a key cluster of molecules that closely related to phagosome formation and maturation by modulating the remodeling of cytoskeleton. Although no direct evidence about phagosome formation and maturation for engulfing and ingesting microorganism was demonstrated in crustacean, to our best knowledge, several important components, mainly small GTPases members such as Rab6, Rab7, RhoA, and Ran, have been reported in some species of shrimp for their involvement in the signaling cascades of the highly conserved processes (51-54). Most of them have also been proven previously to play essential roles in regulating the actin polymerization and dynamic remodeling to form phagosome in vertebrates (51).

Rab7 is a well-known key protein that localizes on the membrane of late endosome and plays a critical role in the phagosome maturation, which has also been found to promote the phagocytosis against $S$. aureus and $V$. parahaemolyticus in hemocytes of E. sinensis (55). However, the role of Rab7, as well as the conversion of Rab5 to Rab7, is still poorly understood in crustaceans. Another Rab family member termed as PjRab6, was found to directly interact with $\beta$-actin, tropomyosin, and the WSSV envelope protein VP466 for generating a complex to regulate hemocytic phagocytosis in $P$. japonicus (56). Interestingly, a further study found that viral envelope protein VP466 was employed by the host cell to increase the GTPase activity of PjRab6 to induce rearrangements of the actin cytoskeleton for the formation of actin stress fibers and subsequently promote the phagocytosis against WSSV (57). All findings indicated that phagocytosis is a complicated process, in particular, phagosome formation and maturation, which not only require host factors but also is associated with the utilization of pathogens components. Moreover, as an important member in phagosome formation and maturation, it is not surprising that PjRab6 could also increase the hemocytic phagocytosis against bacteria, such as $V$. parahaemolyticus, although the regulatory molecular events involved in the phagocytosis of bacteria are still unclear [Figure 1; (54)].

Besides, as one of the Ras GTPase superfamily members, $M j$ RhoA has also been identified in $M$. japonicus to be able to induce the expression level of phagocytosis-related genes ROCK 2 and Arp2/3 as well as to promote the phagocytosis rate against $V$. anguillarum in hemocytes (51). Both ROCK2 and Arp $2 / 3$ were reported to be implicated in the integrinmediated phagocytosis pathway (51). Previously, a C-type lectin receptor, FcLec4, was found to be able to facilitate phagocytosis and bacterial clearance through binding to its receptor $\beta$ integrin in F. chinensis (30). Further study showed that $M j$ RhoA participated in hemocytes phagocytosis against $V$. anguillarum infection via $\beta$-integrin-dependent signaling pathway, which was the downstream factor for $\beta$-integrin (51). Ran GTPases, another family of the small $G$ protein superfamily, has also been found to be involved in phagosome formation and maturation in crustacean hemocytes. For example, PjRan, a Ran GTPase identified from $P$. japonicus, has been proven to modulate hemocytic phagocytosis against WSSV via interaction with the cytoskeleton protein myosin (58). Given the importance and contribution to modulate phagocytosis, researches on exploring more members of the small GTPases family are necessary for understanding the molecular mechanism underlying phagosome formation and maturation against pathogens. Beyond that, the development of phagolysosome also needs to recruit more host components, such as the vacuolar ATPase and NADPH oxidase complex, while various organelles including mitochondria, the endoplasmic reticulum, and Golgi-derived vesicles are also required to make contributions to this complicated physiological process (50). However, extremely poor knowledge is available 


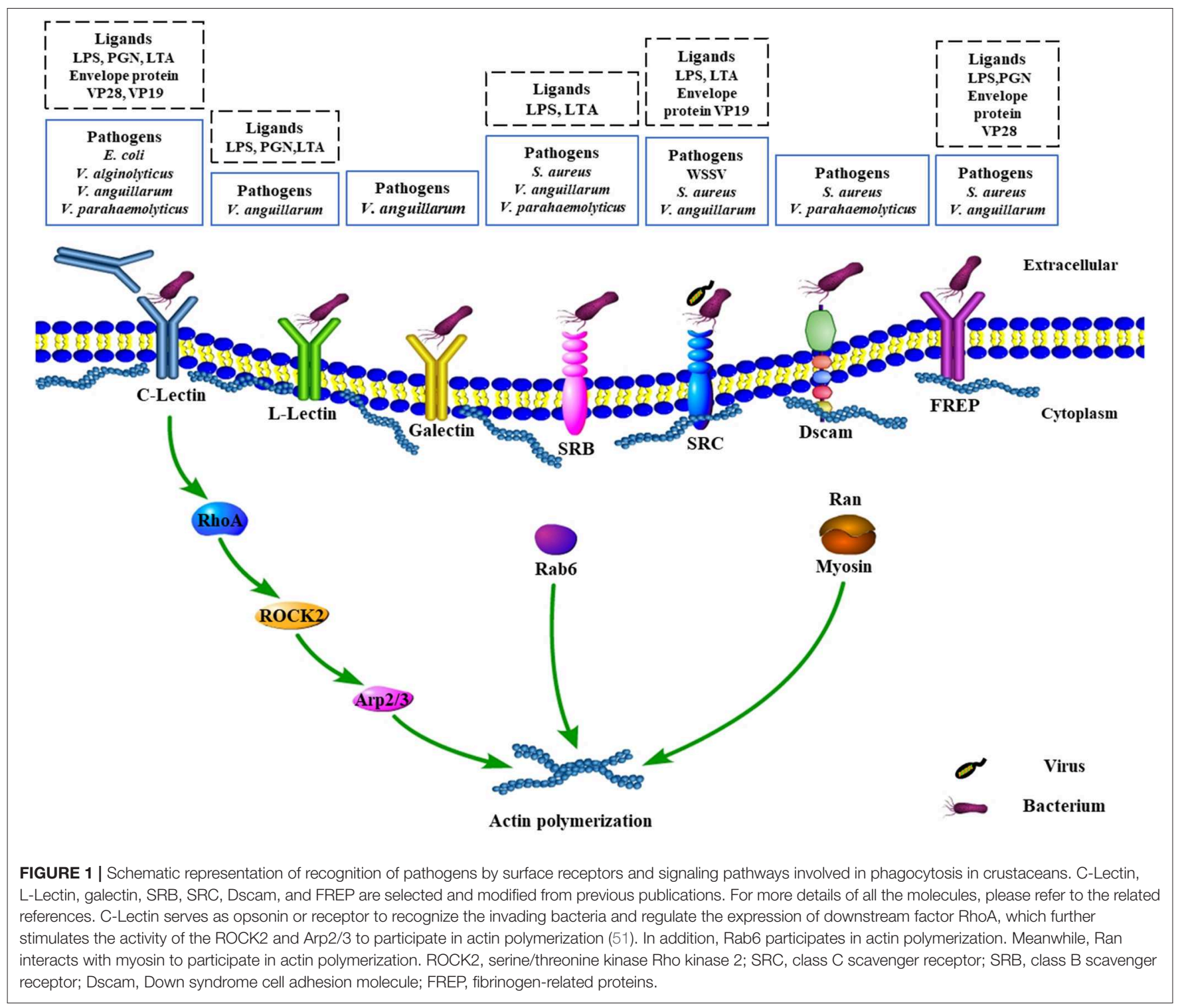

about that due to limited studies conducted in this field in crustaceans.

\section{OTHER FACTORS INVOLVED IN PHAGOCYTOSIS}

Although lots of molecular mechanisms in phagosome formation and maturation have not been well-investigated in crustacean, however, increasing evidence showed that some other factors could also modulate phagocytosis against bacteria. Among them, antimicrobial peptides (AMPs) have been reported as one of the most important components to participate in phagocytosis in crustaceans.

AMPs have been well-characterized as important effectors in the innate immune system in both insect and crustacean, which are mainly expressed in hemocytes and then released into the hemolymph for defensing against a broad spectrum of microorganisms (59). Until now, several types of AMPs, such as penaeidins, antilipopolysaccharide factors, and crustins, have been discovered in crustaceans. Interestingly, in addition to their antimicrobial activity, some AMPs also seem to participate in the phagocytosis of pathogens during microbial infection in shrimp.

Penaeidins are one family of antimicrobial peptides that consist of proline-rich $\mathrm{N}$-terminals and a C-terminal cysteinerich region (60). To date, $\sim 40$ penaeidins have been identified in shrimp, which belong to five types based on their similarity of amino acid sequences. Recently, a penaeidin with an additional serine-rich region ( $M j$ Pen-II) from $M$. japonicus showed not only antimicrobial but also phagocytic activity (61). The rate of phagocytosis was significantly decreased after the MjPen-II gene was silenced, while compensation with injection of $M j P e n-$ II recombinant protein in vivo could increase the phagocytic activity. Further study showed that MjPen-II could eliminate 
bacteria through directly inhibiting bacterial growth as well as promoting phagocytosis.

Crustins are another type of antibacterial peptides that contain a single whey acidic protein domain at the Cterminus. In shrimp, two isoforms of crustins, including MjCru I-1 and crustin-like peptide, have been characterized to be associated with phagocytosis, which were increased in hemocytes after challenge with bacteria (62). Meanwhile, both of them exhibited binding activity to bacteria followed by the increased phagocytosis of hemocyte. In addition, the phagocytic rate of WSSV was significantly decreased when the crustin-like gene was knocked down in shrimp hemocytes, whereas the phagocytic rate of $V$. alginolyticus was increased in hemocytes, exhibiting different biological effect against microorganisms (62). However, the underlying regulatory molecular mechanism of AMP-mediated phagocytosis is still extremely limited, which is worthy of further investigations.

Except AMPs, some other molecules have also been reported to regulate phagocytosis of invading pathogens in crustaceans. For instance, the recombinant phagocytosis-activating protein (PAP) was shown to significantly promote the phagocytic activity of hemocytes in P. monodon (63). Two neuroendocrine factors, crustacean hyperglycemic hormone $(\mathrm{CHH})$ and dopamine, were also found to participate in phagocytosis in L. vannamei hemocytes. $\mathrm{CHH}$ exhibited regulating capacity on phagocytosis through activating nuclear factor kappa B (NF-кB) signaling pathway family members and phagocytosis-related proteins, while dopamine acted as a phagocytosis-related factor to inhibit phagocytosis $(64,65)$. Except for proteins, some microRNAs (miRNAs) have also been found to play critical roles in regulating phagocytosis process in crustaceans. In hemocytes of M. japonicus, miR-1 has been found to negatively regulate the phagocytic activity through interaction with the $3^{\prime}$ untranslated region (UTR) of clathrin heavy chain 1 (CLTC1) gene (66). Moreover, miR-12 and miR-965 were also found to be able to enhance the phagocytosis of WSSV via direct targeting phosphatase and tensin homolog (PTEN) and viral gene wsv240, respectively $(67,68)$, while another miR-100 could promote the antibacterial and antiviral immune response but through regulating the total hemocyte count and phagocytosis in M. japonicus (69). In addition, the shrimp-specific miRS5 acted as a regulator on hemocyte phagocytic progress via negatively regulating myosin expression (70). Taken together, miRNAs act as regulator involved in antibacterial or antiviral immune responses through direct targeting viral genes or cytoskeleton-related genes accordingly. However, the specific

\section{REFERENCES}

1. Vaughan RB. The romantic rationalist: a study of Elie Metchnikoff. Med Hist. (1965) 9:201-15. doi: 10.1017/S0025727300030702

2. Flannagan RS, Jaumouillé V, Grinstein S. The cell biology of phagocytosis. Annu Rev Pathol. (2012) 7:6198. doi: 10.1146/annurev-pathol-011811-132445 regulatory mechanism of miRNA involved in phagocytosis against pathogens needs to be further studied.

\section{CONCLUSION AND FUTURE PERSPECTIVE}

Although several cellular surface receptors/opsonin and intracellular regulator for mediating phagocytosis against bacteria and viruses have been reported in hemocytes from multiple species of crustaceans, limited studies make it extremely unclear until now about the molecular mechanism of recognition of pathogens and the downstream signaling events, in particular those in relation to phagosome formation and maturation, as well as microbe destruction in crustaceans. Future research will be focused on the following fundamental questions in relation to the above concerns. For instance, besides the three described types of hemocytes involved in the phagocytosis in crustaceans, are there any other phagocytic cells types present in crustaceans and additional surface receptors involved in pathogen recognition? What are the regulatory mechanisms for various phagocytic receptors located on hemocytes to cooperate with each other during phagocytosis of different microorganisms? More importantly, are the processes of phagosome formation and maturation in crustaceans similar to that in vertebrates? Last but not least, how does the cooperation occur among various intracellular regulators to mediate phagocytosis, and are there other novel signaling pathways involved in the regulation on hemocyte-mediated phagocytosis? Successfully addressing these important questions will pave avenues to deeper understanding of phagocytosis against microbial infection in crustaceans, in which the fundamental mechanism of phagocytosis in crustacean will benefit the establishment of more efficient control strategies against disease in crustacean farming.

\section{AUTHOR CONTRIBUTIONS}

SL and S-CZ wrote the manuscript. Y-LL contributed to suggestion and discussion. JL and H-PL designed and finally polished the manuscript.

\section{FUNDING}

This work was funded by the National Key Research and Development Program of China (2018YFD0900502), the Natural Science Foundation of China (U1605214, 41676135), and the Fundamental Research Funds for the Central Universities (20720180123). 
determined using a novel genotyping assay. Arch Virol. (2019) 164:206182. doi: 10.1007/s00705-019-04265-2

6. Zheng SC, Xu JY, Liu HP. Cellular entry of white spot syndrome virus and antiviral immunity mediated by cellular receptors in crustaceans. Fish Shellfish Immunol. (2019) 93:580-8. doi: 10.1016/j.fsi.2019.08.011

7. Xiong J, Dai W, Li C. Advances, challenges, and directions in shrimp disease control: the guidelines from an ecological perspective. Appl Microbiol Biotechnol. (2016) 100:6947-54. doi: 10.1007/s00253-016-7679-1

8. Gordon S. Phagocytosis: an immunobiologic process. Immunity. (2016) 44:463-75. doi: 10.1016/j.immuni.2016.02.026

9. Johansson MW, Keyser P, Sritunyalucksana K, Söderhäll K. Crustacean haemocytes and haematopoiesis. Aquaculture. (2000) 191:45-52. doi: 10.1016/S0044-8486(00)00418-X

10. Battison A, Cawthorn R, Horney B. Classification of Homarus americanus hemocytes and the use of differential hemocyte counts in lobsters infected with Aerococcus viridans var. homari (Gaffkemia). J Invertebr Pathol. (2003) 84:177-97. doi: 10.1016/j.jip.2003.11.005

11. Söderhäll I. Crustacean hematopoiesis. Dev Comp Immunol. (2016) 58:12941. doi: 10.1016/j.dci.2015.12.009

12. Wu L, Söderhäll I, Kim YA, Liu HP, Söderhäll K. Hemocyte-lineage marker proteins in a crustacean, the freshwater crayfish, Pacifastacus leniusculus. Proteomics. (2008) 8:4226-35. doi: 10.1002/pmic.200800177

13. Lin X, Söderhäll I. Crustacean hematopoiesis and the astakine cytokines. Blood. (2011) 117:6417-24. doi: 10.1182/blood-2010-11-320614

14. Lavine MD, Strand MR. Insect hemocytes and their role in immunity. Insect Biochem Mol Biol. (2002) 32:1295-309. doi: 10.1016/S0965-1748(02)00092-9

15. Zhou YL, Gu WB, Tu DD, Zhu QH, Zhou ZK, Chen YY, et al. Hemocytes of the mud crab Scylla paramamosain: Cytometric, morphological characterization and involvement in immune responses. Fish Shellfish Immunol. (2017) 72:459-69. doi: 10.1016/j.fsi.2017.10.055

16. Lv S, Xu J, Zhao J, Yin N, Lu B, Li S, et al. Classification and phagocytosis of circulating haemocytes in Chinese mitten crab (Eriocheir sinensis) and the effect of extrinsic stimulation on circulating haemocytes in vivo. Fish Shellfish Immunol. (2014) 39:415-22. doi: 10.1016/j.fsi.2014.05.036

17. Sang HM, Fotedar R. Effects of mannan oligosaccharide dietary supplementation on performances of the tropical spiny lobsters juvenile (Panulirus ornatus, Fabricius 1798). Fish Shellfish Immunol. (2010) 28:483-9. doi: 10.1016/j.fsi.2009.12.011

18. Sung HH, Hwang SF, Tasi FM. Responses of giant freshwater prawn (Macrobrachium rosenbergii) to challenge by two strains of Aeromonas spp. J Invertebr Pathol. (2000) 76:278-84. doi: 10.1006/jipa.2000.4981

19. Chai YM, Zhu Q, Yu SS, Zhao XF, Wang JX. A novel protein with a fibrinogen-like domain involved in the innate immune response of Marsupenaeus japonicus. Fish Shellish Immunol. (2012) 32:307-15. doi: 10.1016/j.fsi.2011.11.020

20. Li F, Chang X, Xu L, Yang F. Different roles of crayfish hemocytes in the uptake of foreign particles. Fish Shellfish Immunol. (2018) 77:1129. doi: 10.1016/j.fsi.2018.03.029

21. Wang XW, Wang JX. Diversity and multiple functions of lectins in shrimp immunity. Dev Comp Immunol. (2013) 39:27-38. doi: 10.1016/j.dci.2012.04.009

22. Canton J, Neculai D, Grinstein S. Scavenger receptors in homeostasis and immunity. Nat Rev Immunol. (2013) 13:621-34. doi: 10.1038/nri3515

23. Dong Y, Dimopoulos G. Anopheles fibrinogen-related proteins provide expanded pattern recognition capacity against bacteria and malaria parasites. J Biol Chem. (2009) 284:9835-44. doi: 10.1074/jbc.M807084200

24. Dong Y, Taylor HE, Dimopoulos G. AgDscam, a hypervariable immunoglobulin domain-containing receptor of the Anopheles gambiae innate immune system. PLoS Biol. (2006) 4:e229. doi: 10.1371/journal.pbio.0040229

25. Luo $\mathrm{T}$, Yang HF, Zhang $\mathrm{X}, \mathrm{Xu} \mathrm{X}$. Purification, characterization and cDNA cloning of a novel lipopolysaccharide-binding lectin from the shrimp Penaeus monodon. Dev Comp Immunol. (2006) 30:607-17. doi: 10.1016/j.dci.2005.10.004

26. Zhang X, Pan L, Yu J, Huang H. One recombinant C-type lectin ( $L v$ Lec) from white shrimp Litopenaeus vannamei affected the haemocyte immune response in vitro. Fish Shellfish Immunol. (2019) 89:35-42. doi: 10.1016/j.fsi.2019.03.029
27. Luo M, Yang L, Wang ZA, Zuo H, Weng S, He J, et al. A novel C-type lectin with microbiostatic and immune regulatory functions from Litopenaeus vannamei. Fish Shellish Immunol. (2019) 93:3618. doi: 10.1016/j.fsi.2019.07.047

28. Song F, Chen GL, Lu KC, Fan JQ, Yan MT, He HH, et al. Identification and functional characterization of a C-type lectin gene from Litopenaeus vannamei that is associated with ER-stress response. Fish Shellfish Immunol. (2019) 93:977-85. doi: 10.1016/j.fsi.2019.08.056

29. Liang ZW, Yang LW, Zheng JF, Zuo HL, Weng SP, He JG, et al. A lowdensity lipoprotein receptor (LDLR) class a domain-containing C-type lectin from Litopenaeus vannamei plays opposite roles in antibacterial and antiviral responses. Dev Comp Immunol. (2019) 92:29-34. doi: 10.1016/j.dci.2018. 11.002

30. Wang XW, Zhao XF, Wang JX. C-type lectin binds to beta integrin to promote hemocytic phagocytosis in shrimp. J Biol Chem. (2014) 289:240514. doi: $10.1074 /$ jbc.M113.528885

31. Wei X, Wang L, Sun W, Zhang M, Ma H, Zhang Y, et al. C-type lectin B $(S p C T L-B)$ regulates the expression of antimicrobial peptides and promotes phagocytosis in mud crab Scylla paramamosain. Dev Comp Immunol. (2018) 84:213-29. doi: 10.1016/j.dci.2018.02.016

32. Zhang XW, Wang Y, Wang XW, Wang L, Mu Y, Wang JX. A C-type lectin with an immunoglobulin-like domain promotes phagocytosis of hemocytes in crayfish Procambarus clarkii. Sci Rep. (2016) 6:29924. doi: 10.1038/srep29924

33. Chen DD, Meng XL, Xu JP, Yu JY, Meng MX, Wang J. PcLT, a novel C-type lectin from Procambarus clarkii, is involved in the innate defense against Vibrio alginolyticus and WSSV. Dev Comp Immunol. (2013) 39:25564. doi: $10.1016 /$ j.dci.2012.10.003

34. Sen X, Lei W, Xian-Wei W, Yan-Ran Z, Wen-Jie B, Xiao-Fan Z, et al. L-Type lectin from the kuruma shrimp Marsupenaeus japonicus promotes hemocyte phagocytosis. Dev Comp Immunol. (2014) 44:397405. doi: 10.1016/j.dci.2014.01.016

35. Hou FJ, Liu YJ, He SL, Wang XZ, Mao AT, Liu ZG, et al. A galectin from shrimp Litopenaeus vannamei is involved in immune recognition and bacteria phagocytosis. Fish Shellfish Immunol. (2015) 44:58491. doi: $10.1016 /$ j.fsi.2015.03.017

36. Kong T, Gong Y, Liu Y, Wen X, Tran NT, Aweya JJ, et al. Scavenger receptor $B$ promotes bacteria clearance by enhancing phagocytosis and attenuates white spot syndrome virus proliferation in Scylla paramamosian. Fish Shellfish Immunol. (2018) 78:79-90. doi: 10.1016/j.fsi.2018.04.027

37. Yang MC, Shi XZ, Yang HT, Sun JJ, Xu L, Wang XW, et al. Wang. Scavenger receptor C mediates phagocytosis of white spot syndrome virus and restricts virus proliferation in shrimp. PLoS Pathog. (2016) 12:e1006127. doi: 10.1371/journal.ppat.1006127

38. Yang MC, Yang HT, Li J, Sun JJ, Bi WJ, Niu GJ, et al. Scavenger receptor C promotes bacterial clearance in kuruma shrimp Marsupenaeus japonicus by enhancing hemocyte phagocytosis and AMP expression. Fish Shellfish Immunol. (2017) 67:254-62. doi: 10.1016/j.fsi.2017. 06.003

39. Bi WJ, Li DX, Xu YH, Xu S, Li J, Zhao XF, et al. Scavenger receptor B protects shrimp from bacteria by enhancing phagocytosis and regulating expression of antimicrobial peptides. Dev Comp Immunol. (2015) 51:1021. doi: 10.1016/j.dci.2015.02.001

40. Wu YM, Yang L, Li XJ, Li L, Wang Q, Li WW. A class B scavenger receptor from Eriocheir sinensis (EsSR-B1) restricts bacteria proliferation by promoting phagocytosis. Fish Shellfish Immunol. (2017) 70:426-36. doi: 10.1016/j.fsi.2017.09.034

41. Li XJ, Yang L, Li D, Zhu YT, Wang Q, Li WW. Pathogen-specific binding soluble down syndrome cell adhesion molecule (Dscam) regulates phagocytosis via membrane-bound Dscam in crab. Front Immunol. (2018) 9:801. doi: 10.3389/fimmu.2018.00801

42. Sun JJ, Lan JF, Shi XZ, Yang MC, Yang HT, Zhao XF, et al. A fibrinogen-related protein (FREP) is involved in the antibacterial immunity of Marsupenaeus japonicus. Fish Shellfish Immunol. (2014) 39:296-304. doi: 10.1016/j.fsi.2014.05.005

43. Lu Y, Su F, Li Q, Zhang J, Li Y, Tang T, et al. Pattern recognition receptors in Drosophila immune responses. Dev Comp Immunol. (2020) 102:103468. doi: 10.1016/j.dci.2019.103468 
44. Watson FL, Puttmann-Holgado R, Thomas F, Lamar DL, Hughes M, Kondo $\mathrm{M}$, et al. Extensive diversity of Ig-superfamily proteins in the immune system of insects. Science. (2005) 309:1874-78. doi: 10.1126/science.1116887

45. Li D, Yu AQ, Li XJ, Zhu YT, Jin XK, Li WW, et al. Antimicrobial activity of a novel hypervariable immunoglobulin domain-containing receptor Dscam in Cherax quadricarinatus. Fish Shellfish Immunol. (2015) 47:76676. doi: 10.1016/j.fsi.2015.10.025

46. Chou PH, Chang HS, Chen IT, Lee CW, Hung HY, Han-Ching Wang KC. Penaeus monodon Dscam (PmDscam) has a highly diverse cytoplasmic tail and is the first membrane-bound shrimp Dscam to be reported. Fish Shellfish Immunol. (2011) 30:1109-23. doi: 10.1016/j.fsi.2011.02.009

47. Chou PH, Chang HS, Chen IT, Lin HY, Chen YM, Yang HL, et al. The putative invertebrate adaptive immune protein Litopenaeus vannamei Dscam ( $L v$ Dscam) is the first reported Dscam to lack a transmembrane domain and cytoplasmic tail. Dev Comp Immunol. (2009) 33:125867. doi: 10.1016/j.dci.2009.07.006

48. Wang J, Yang B, Wang W, Song X, Jiang Q, Qiu L, et al. The enhanced immune protection in Chinese mitten crab Eriocheir sinensis against the second exposure to bacteria Aeromonas hydrophila. Front Immunol. (2019) 10:2041. doi: 10.3389/fimmu.2019.02041

49. Wang X, Zhao Q, Christensen BM. Identification and characterization of the fibrinogen-like domain of fibrinogen-related proteins in the mosquito, Anopheles gambiae, and the fruitfly, Drosophila melanogaster, genomes. BMC Genomics. (2005) 6:114. doi: 10.1186/1471-2164-6-114

50. Pauwels M, Trost M, Beyaert R, Hoffmann E. Patterns, receptors, and signals: regulation of phagosome maturation. Trends Immunol. (2017) 38:40722. doi: 10.1016/j.it.2017.03.006

51. Xu JD, Diao MQ, Niu GJ, Wang XW, Zhao XF, Wang JX. A small GTPase, RhoA, inhibits bacterial infection through integrin mediated phagocytosis in invertebrates. Front Immunol. (2018) 9:1928. doi: 10.3389/fimmu.2018.01928

52. Sayedyahossein S, Dagnino L. Integrins and small GTPases as modulators of phagocytosis. Int Rev Cel Mol Biol. (2013) 302:321-54. doi: 10.1016/B978-0-12-407699-0.00006-6

53. Zhao Z, Jiang CX, Zhang XB. Effects of immunostimulants targeting Ran GTPase on phagocytosis against virus infection in shrimp. Fish Shellfish Immunol. (2011) 31:1013-8. doi: 10.1016/j.fsi.2011.08.022

54. Zong RR, Wu WL, Xu JY, Zhang XB. Regulation of phagocytosis against bacterium by Rab GTPase in shrimp Marsupenaeus japonicus. Fish Shellfish Immunol. (2008) 25:258-63. doi: 10.1016/j.fsi.2008.05.006

55. Wang Q, Li H, Zhou K, Qin X, Wang Q, Li W. Rab7 controls innate immunity by regulating phagocytosis and antimicrobial peptide expression in Chinese mitten crab. Fish Shellfish Immunol. (2019) 95:25967. doi: 10.1016/j.fsi.2019.10.037

56. Wu WL, Zong RR, Xu JY, Zhang XB. Antiviral phagocytosis is regulated by a novel rab-dependent complex in shrimp Penaeus japonicus. J Proteome Res. (2008) 7:424-31. doi: 10.1021/pr700639t

57. Ye $\mathrm{T}$, Zong $\mathrm{R}$, Zhang $\mathrm{X}$. The role of white spot syndrome virus (WSSV) VP466 protein in shrimp antiviral phagocytosis. Fish Shellfish Immunol. (2012) 33:350-8. doi: 10.1016/j.fsi.2012.05.017

58. Liu WF, Han F, Zhang XB. Ran GTPase regulates hemocytic phagocytosis of shrimp by interaction with myosin. J Proteome Res. (2009) 8:1198206. doi: $10.1021 /$ pr800840x
59. Tassanakajon A, Amparyup P, Somboonwiwat K, Supungul P. Cationic antimicrobial peptides in penaeid shrimp. Mar Biotechnol. (2011) 13:63957. doi: 10.1007/s10126-011-9381-8

60. Vaseeharan B, Shanthi S, Chen JC, Espineira M. Molecular cloning, sequence analysis and expression of Fein-Penaeidin from the haemocytes of Indian white shrimp Fenneropenaeus indicus. Results Immunol. (2012) 2:35-43. doi: 10.1016/j.rinim.2012.02.001

61. An MY, Gao J, Zhao XF, Wang JX. A new subfamily of penaeidin with an additional serine-rich region from kuruma shrimp (Marsupenaeus japonicus) contributes to antimicrobial and phagocytic activities. Dev Comp Immunol. (2016) 59:186-98. doi: 10.1016/j.dci.2016.02.001

62. Sun B, Wang Z, Zhu F. The crustin-like peptide plays opposite role in shrimp immune response to Vibrio alginolyticus and white spot syndrome virus (WSSV) infection. Fish Shellfish Immunol. (2017) 66:48796. doi: 10.1016/j.fsi.2017.05.055

63. Deachamag P, Intaraphad U, Phongdara A, Chotigeat W. Expression of a phagocytosis activating protein (PAP) gene in immunized black tiger shrimp. Aquaculture (2006) 255:165-72. doi: 10.1016/j.aquaculture.2006.01.010

64. Xu L, Pan L, Zhang X, Wei C. Effects of crustacean hyperglycemic hormone $(\mathrm{CHH})$ on regulation of hemocyte intracellular signaling pathways and phagocytosis in white shrimp Litopenaeus vannamei. Fish Shellfish Immunol. (2019) 93:559-66. doi: 10.1016/j.fsi.2019.07.051

65. Tong R, Wei C, Pan L, Zhang X. Effects of dopamine on immune signaling pathway factors, phagocytosis and exocytosis in hemocytes of Litopenaeus vannamei. Dev Comp Immunol. (2019) 102:103473. doi: 10.1016/j.dci.2019.103473

66. Liu C, Wang J, Zhang $X$. The involvement of MiR-1-clathrin pathway in the regulation of phagocytosis. PLoS ONE. (2014) 9:e98747. doi: 10.1371/journal.pone.0098747

67. Shu L, Zhang X. Shrimp miR-12 suppresses white spot syndrome virus infection by synchronously triggering antiviral phagocytosis and apoptosis pathways. Front Immunol. (2017) 8:855. doi: 10.3389/fimmu.2017.00855

68. Shu L, Li CR, Zhang XB. The role of shrimp miR-965 in virus infection. Fish Shellfish Immunol. (2016) 54:427-34. doi: 10.1016/j.fsi.2016.04.129

69. Wang Z, Zhu F. MicroRNA-100 is involved in shrimp immune response to white spot syndrome virus (WSSV) and Vibrio alginolyticus infection. Sci Rep. (2017) 7:42334. doi: 10.1038/srep42334

70. Wang Z, Zhu F. Different roles of a novel shrimp microRNA in white spot syndrome virus (WSSV) and Vibrio alginolyticus infection. Dev Comp Immunol. (2018) 79:21-30. doi: 10.1016/j.dci.2017. 10.002

Conflict of Interest: The authors declare that the research was conducted in the absence of any commercial or financial relationships that could be construed as a potential conflict of interest.

Copyright (C) $2020 \mathrm{Liu}$, Zheng, Li, Li and Liu. This is an open-access article distributed under the terms of the Creative Commons Attribution License (CC BY). The use, distribution or reproduction in other forums is permitted, provided the original author(s) and the copyright owner(s) are credited and that the original publication in this journal is cited, in accordance with accepted academic practice. No use, distribution or reproduction is permitted which does not comply with these terms. 